\title{
Enfermagem e idoso: grupos de pesquisa cadastrados no diretório do $\mathrm{CNPq}$
}

Recebido em 07/12/2012

Aprovado em: 27/07/2013

\author{
Silvana Sidney da Costa Santos ${ }^{1}$ \\ Danielle Adriane Silveira Vidal ${ }^{2}$ \\ Jamila Geri Tomaschewski Barlem ${ }^{3}$ \\ Karine Gracinda da Silva Xavier ${ }^{4}$ \\ Diéssica Roggia Piexak ${ }^{5}$ \\ Daiane Porto Gautério ${ }^{6}$
}

Resumo: Teve-se como objetivo analisar os grupos de estudo e pesquisa, da Enfermagem, cadastrados no Diretório dos Grupos de Pesquisa do Conselho Nacional de Desenvolvimento Científico e Tecnológico, com linha de pesquisa com ênfase no idoso. Pesquisa documental realizada entre agosto e outubro de 2011, com análise estatística descritiva, mediante cálculos de frequência. Identificados 51 grupos, com a maior concentração nas regiões Nordeste, Sudeste e Sul. Vislumbra-se a necessidade de estudos voltados ao idoso e a relevância da participação do enfermeiro em grupos de estudo e pesquisa, contribuindo para o fortalecimento e na busca de novos conhecimentos na temática investigada.

Palavras-Chave: Idoso. Grupos de Pesquisa. Pesquisa em Enfermagem. Enfermagem.

\section{Nursing and elderly: research group registered in the directory of CNPq}

Abstract: The aim of this study was to analyze the study and research groups, of the nursing area, registered in the Directory of Research Groups of the National Council to Development Scientific and Technological, which have a line of research with emphasis on the elderly. This is a documentary research, held between August and October 2011, the analysis occurred by descriptive statistics, by frequency calculations. Were identified 51 groups, with highest concentration in the northeast, southeast and south. Glimpses the need of studies directed the elderly and the importance of the nurses participation in the study and research groups, which can contribute to strengthening and search for new knowledge in the subject investigated. Keywords: Aged. Research Groups. Nursing Research. Nursing.

\section{Enfermería y ancianos: grupo de investigación registrados en el directorio del CNPq}

Resumen: El objectivo fue analizar los grupos de estúdio e investigación del área de enfermería, registrados en el directorio de Grupos de Investigación del Consejo Nacional de Desarrollo Científico y Tecnológico, que tienen uma línea de investigación con énfasis en los ancianos. Investigación documental realizada entre agosto y octubre de 2011, cuyo análisis fue mediante estadística descriptiva por medio de cálculos de frecuencia. Se identificaron 51 grupos con la mayor concentración en El Nordeste, Sudeste y Sur. Se vislumbra de la necesidad de estúdios a los ancianos y la importancia de la participación de las enfermeras en los grupos de estudio e investigación, que puede contribuir al fortalecimiento y la búsqueda de nuevos conocimientos en el tema investigado.

Palabras Clave: Anciano. Grupos de Investigación. Investigación en Enfermería. Enfermería.

\section{INTRODUÇÃO}

$\mathrm{D}$ e modo a conhecer os elementos que permeiam os grupos de estudo e pesquisa com ênfase na temática a pessoa idosa, na área da Enfermagem, realizou-se uma pesquisa documental no Diretório de Grupos de Pesquisa do Conselho Nacional de Desenvolvimento Científico e Tecnológico (CNPq).

$O$ interesse dos enfermeiros pela população idosa intensificou-se a partir da ocorrência da transição demográfica, da constituição de leis específicas instituídas para garantir os direitos dos idosos e do incentivo para difundir o ensino de Geriatria e Gerontologia nos cursos universitários, repercutindo no aumento da produção científica em Enfermagem nessa temática ${ }^{(1)}$.

A pesquisa em Enfermagem Gerontogeriátrica foi impulsionada pelo aumento do envelhecimento populacional e pela preocupação dos enfermeiros em compreender as necessidades das pessoas idosas, com o objetivo de possibilitarem-Ihes um cuidado específico e qualificado ${ }^{(2)}$.
No Brasil, a produção científica em pesquisa vem sendo desenvolvida por investigadores titulados e/ou estudantes em nível de treinamento, organizados sob a forma de Grupos de Estudo e Pesquisa. Na Enfermagem, esses grupos surgiram a partir da década de 1970, expandindo-se de forma significativa ao longo dos anos, representando aproximadamente 323 grupos cadastrados no Diretório de Grupos de Estudo e Pesquisa do $\mathrm{CNPq}^{(3)}$.

Os estudos brasileiros acerca do envelhecimento, na área da Enfermagem, iniciaram na década de 1970, com algumas produções dispersas e com a criação do Núcleo de Estudos e Pesquisa do Idoso (NESPI) da Universidade Federal da Bahia, em 1973, primeiro Grupo de Pesquisa em Enfermagem com ênfase na população idosa ${ }^{(4)}$.

No decorrer das décadas de 1970 e 1980, a Enfermagem destacou-se pelo seu pioneirismo na formação de grupos de estudo e pesquisa específicos ao estudo das pessoas idosas,

'Enfermeira. Doutora em Enfermagem. Docente da Escola de Enfermagem (EEnf) - Universidade Federal do Rio Grande (FURG). Rio Grande - RS. Brasil.

${ }^{2}$ Enfermeira. Mestranda do Programa de Pós-Graduação em Enfermagem (PPGEnf) -FURG. Bolsista de Mestrado do CNPq. Rio Grande - RS. Brasil.

${ }^{3}$ Enfermeira. Doutoranda do PPGEnf -FURG. Bolsista de Doutorado da FAPERGS. Rio Grande - RS. Brasil.

${ }^{4}$ Enfermeira. Mestranda do PPGEnf-FURG. Rio Grande - RS. Brasil.

${ }^{5}$ Enfermeira. Doutoranda do PPGEnf-FURG. Bolsista CAPES/REUNI. Rio Grande - RS. Brasil.

${ }^{6}$ Enfermeira. Doutoranda do PPGEnf-FURG. Bolsista CAPES/REUNIRio Grande - RS. Brasil. 
uma vez que nesse período, dos quatro grupos de pesquisa existentes no Brasil, três eram da área da enfermagem, o que contribuiu para a construção da história da Gerontologia no Brasil $^{(1)}$.

A análise dos grupos de estudo e pesquisa em Enfermagem direcionados ao idoso, que retratam o interesse pelo processo de envelhecimento humano, torna-se uma estratégia imprescindível para a compreensão da evolução do conhecimento científico em Enfermagem Gerontogeriátrica no Brasil. A pesquisa em Enfermagem Gerontogeriátrica evidencia a trajetória percorrida pela comunidade acadêmica na construção de conhecimentos específicos e fundamentada, contribuindo para um cuidado específico ao idoso e refletindo na visibilidade da profissão1.

Foram questionamentos deste estudo: quantos são os grupos de estudo e pesquisa que abordam o idoso, no Diretório dos Grupos de Pesquisa do CNPq, Brasil? Quem e quantos são os pesquisadores e estudantes da temática na Enfermagem? Em quê Regiões e Estados se localizam esses Grupos? Como se caracterizam as linhas de pesquisa com ênfase no idoso?

Partindo destes questionamentos, o objetivo deste estudo foi analisar os grupos de estudo e pesquisa, da área da Enfermagem, cadastrados no Diretório dos Grupos de Pesquisa do Conselho Nacional de Desenvolvimento Científico e Tecnológico, que possuem linha de pesquisa com ênfase no idoso.

\section{METODOLOGIA}

Pesquisa documental, que se utiliza de métodos e técnicas para a apreensão, compreensão e análise de documentos dos mais variados tipos ${ }^{(5)}$. O documento como fonte de pesquisa pode ser escrito e não escrito, tais como filmes, vídeos, slides, fotografias ou pôsteres. Esses documentos são utilizados como fontes de informações, indicações e esclarecimentos que trazem seu conteúdo para elucidar determinadas questões e servir de prova para outras, de acordo com o interesse do pesquisador ${ }^{(6)}$.

A pesquisa documental foi realizada por meio de levantamento de dados dos grupos de pesquisa do Brasil, cadastrados no Diretório dos Grupos de Pesquisa CNPq, utilizando-se a palavra chave idoso em sua base corrente, em agosto de 2011 . O Diretório dos Grupos de Pesquisa é um projeto desenvolvido pelo CNPq desde 1992, constituindo-se em bases de dados que possibilitam o acesso às informações acerca dos grupos de pesquisa em atividade no $\mathrm{Brasil}^{(7)}$.

Para a coleta desta pesquisa documental foram realizados os seguintes passos: 1 . Acesso ao site de busca de informações do Diretório dos Grupos de Pesquisa no Brasil (http://dgp. cnpq.br/buscaoperacional/); 2 . Em seguida, foi selecionada a opção Grupos e inserida na caixa de pesquisa a palavra chave idoso; 3. No filtro de busca foi selecionada a área do Grupo Enfermagem.

A partir desses passos, foi possível identificar 62 Grupos de Pesquisa em Enfermagem do Brasil, relacionados ao tema idoso, o que oportunizou o acesso as informações de cada grupo. Foi considerado como critério de inclusão: possuir pelo menos uma linha de pesquisa com ênfase no tema idoso. Dos 62 grupos identificados, foram excluídos 11 por não apresentarem ênfase na temática idoso descrita nos objetivos das linhas de pesquisa ou na descrição da linha. A amostra deste estudo totalizou 51 grupos.

Apresentar uma linha de pesquisa indica que os estudos originados pelo grupo foquem uma temática central, por isso os resultados obtidos por meio dessas investigações são congruentes e complementares ${ }^{(7)}$.

Após a seleção dos 51 grupos, as informações foram coletadas tendo em vista os questionamentos do estudo.

Os dados coletados foram tabulados e organizados no Excel 2007, de acordo com: distribuição por Estado; ano de formação do Grupo de Pesquisa; instituição a qual pertence; número de integrantes, identificados quanto ao status de pesquisadores, estudantes e técnicos. A análise dos dados foi realizada por meio de estatística descritiva mediante cálculos de frequência. Foram construídos gráficos e tabelas, que permitiram melhor visualização dos resultados e a sua interpretação.

O presente estudo não necessitou ser submetido a um comitê de ética em pesquisa, por tratar-se de uma investigação documental, e ter utilizado fontes de domínio público para a realização. Foram seguidos rigorosamente os cuidados éticos na busca, análise e discussão e apresentação dos resultados.

\section{RESULTADOS}

A partir dos dados coletados no Diretório dos Grupos de Pesquisa do CNPq foram identificados 51 grupos de estudo e pesquisa, na área da Enfermagem, com ênfase no idoso, distribuídos em 39 instituições de ensino superior, públicas e particulares. As instituições de ensino superior que apresentaram maior número de grupos de estudo e pesquisa, correspondendo três grupos, foram: Universidade de São Paulo (USP), Universidade Federal do Maranhão (UFMA) e Universidade Federal da Bahia (UFBA).

Os grupos de pesquisa analisados foram fundados entre os anos de 1973 e 2010.

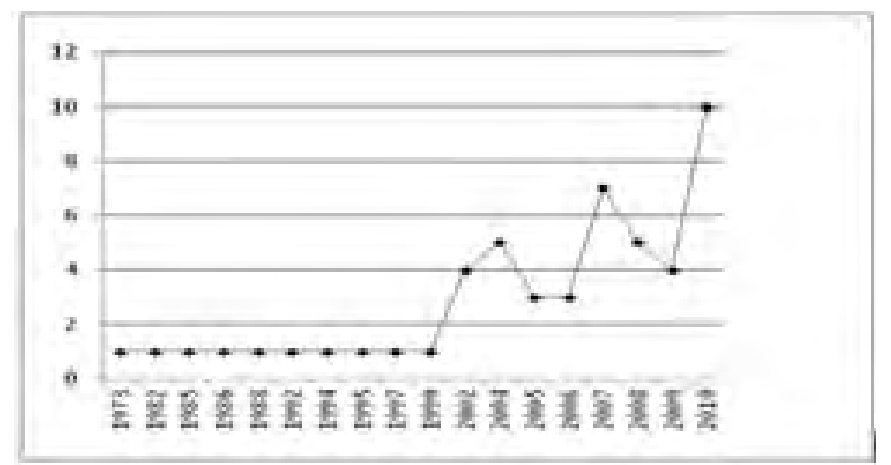

Figura 1. Número de grupos de pesquisa em enfermagem com linha de pesquisa com ênfase no idoso, em relação ao ano de sua função, 2011.

Com relação à distribuição dos grupos de estudo e pesquisa, com foco no idoso, por regiões foi verificado: 16 no Nordeste, 15 no Sudeste, 11 no Sul, seis no Centro-Oeste e três no Norte.

Quando verificada essa relação às unidades federadas observou-se que São Paulo concentra o maior número, com 10 grupos, seguindo pelo Rio Grande do Sul, com a segunda maior concentração, com cinco grupos. 


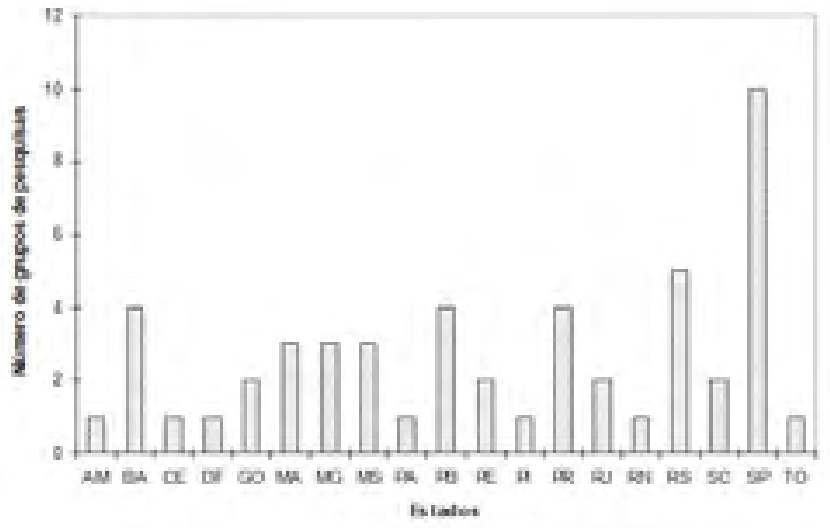

Figura 2. Grupos de pesquisa em enfermagem com linha de pesquisa com ênfase no idoso, por Estado em 2011.

No que tange ao número de linhas de pesquisa, evidenciou-se a presença de 181 linhas distribuídas nos 51 grupos investigados. Alguns grupos apresentaram até 14 linhas de pesquisa. Destaca-se que 32 grupos apresentam de uma a três linhas de pesquisa; 15, de quatro a seis; quatro, de sete a 14.

Dentre as linhas de pesquisa dos grupos analisados evidenciou-se que 26 dessas linhas estavam relacionadas à saúde do idoso e 12 relacionavam-se com o cuidado ao idoso e as demais com outras temáticas: aumento da expectativa de vida; direcionamento do cuidado ao idoso, reconhecendo especificidades e estimulando a independência e autonomia.

Foram identificados 1319 integrantes nos grupos pesquisados. Entre eles 460 eram pesquisadores, 729 estudantes e 130 técnicos. Verificou-se que entre os 460 pesquisadores cadastrados nos grupos de estudo e pesquisa, 234 eram doutores; 140 mestres; 46 especialistas; 14 graduados. Destes pesquisadores, 16 possuem bolsa de produtividade do CNPq, conforme ilustrado no gráfico a seguir.

Em relação aos 731 estudantes cadastrados nos grupos de estudo e pesquisa, da área da Enfermagem, com ênfase no idoso, 78 estavam cursando o doutorado; 151 eram estudantes do mestrado; 80 realizavam especialização; 375 eram da graduação, sete de nível médio e 40 não possuíam informações no currículo Lattes.

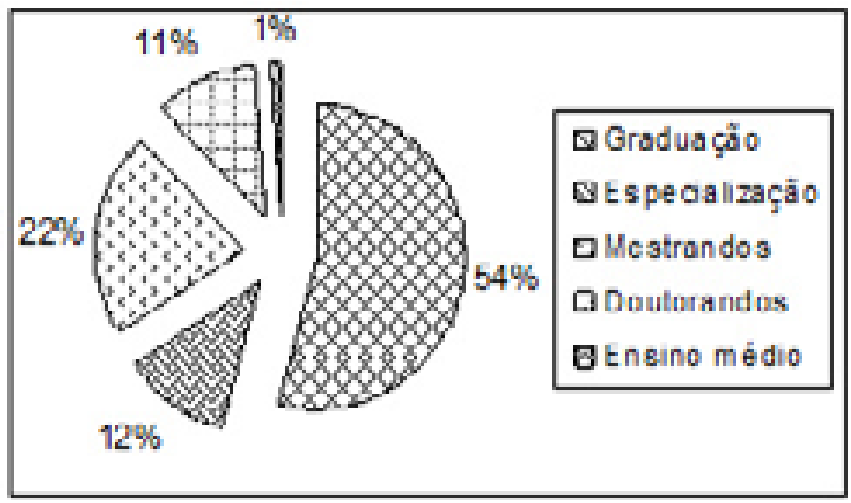

Figura 3. Formação dos estudantes nos grupos de pesquisa em enfermagem com linha de pesquisa com ênfase no idoso, 2011

Evidenciou-se a participação de pesquisadores de outras áreas do conhecimento, como Medicina, Psicologia, Fisioterapia, Gerontologia, Terapia ocupacional, Direito, História e Letras, o que contribui para construção do conhecimento interdisciplinar.

\section{DISCUSSÃO}

O aumento dos programas de pós-graduação e a elevação na titulação dos docentes têm trazido um grande crescimento no número de grupos de estudo e pesquisa e contribuído na produção de conhecimento, fornecendo, nas últimas décadas, um importante desenvolvimento científico brasileiro ${ }^{(8)}$, principalmente na ciência em formação: Enfermagem.

Os grupos de pesquisa, os quais estão fortemente vinculados aos programas de pós-graduação, possibilitam produções científicas, fortalecem a formação de profissionais mais qualificados, além de contribuírem para a consolidação da Enfermagem como uma profissão e ciência em formação ${ }^{(9)}$.

As Regiões Nordeste, Sudeste e Sul descaram-se em números de grupos de estudo e pesquisa na área da enfermagem, com ênfase no idoso. Esses dados demonstram que à medida que a pós-graduação em Enfermagem no Brasil desenvolve-se, aumentam-se esses grupos ${ }^{(10)}$.

A Região Sudeste do Brasil foi à primeira há possuir um Programa de Pós-Graduação Stricto Sensu em Enfermagem o que contribuiu para o aperfeiçoamento do enfermeiro para a docência, assistência e pesquisa. Essa região apresenta uma estrutura sócio-econômica que favorece o desenvolvimento científico e tecnológico, devido aos incentivos políticos e aos níveis de industrialização elevados que possibilitam fundos financeiros para a Região e força político-social das instituições de ensino ${ }^{(9)}$.

Na Região Norte o número de programas de pós-graduação em Enfermagem corresponde a dois, localizados em Belém, Pará. A Universidade Federal do Pará (UFPA) e a Universidade Estadual do Pará (UEPA) são as primeiras universidades da região Norte a oferecer mestrado em Enfermagem ${ }^{(11)}$. Esses dados evidenciam as desigualdades entre as regiões do Brasil em relação aos programas de pós-graduação em Enfermagem e o reduzido ainda, número de grupos de pesquisa.

São Paulo e o Rio Grande do Sul concentram o maior número de grupos de estudo e pesquisa na área da enfermagem, com ênfase no idoso, em relação aos demais Estados. Essa situação é constata devido ao maior número de programas de pós-graduação em Enfermagem nas Regiões Sudeste e Sul(11).

Conforme o Censo de 2010 do Instituto Brasileiro de Geografia e Estatística (IBGE), o Rio Grande do Sul é o Estado que apresenta a maior população de idosos ${ }^{(12)}$. Os enfermeiros pesquisadores do Estado do Rio Grande do Sul visualizaram essa demanda e perceberam a necessidade de desenvolvimento de grupos de estudo e pesquisa específicos para área do conhecimento na temática idoso.

Destacam-se como motivos de pesquisa e reflexões acerca do idoso: o crescente aumento da expectativa de vida e consequente envelhecimento populacional que influenciam e mudam as necessidades de saúde ${ }^{(13)}$; os desafios da saúde para diminuir as iatrogenias nos idosos e a necessidade de cuidado especializado para essas pessoas ${ }^{(14)}$; a necessidade da contribuição dos profissionais da saúde para a racionalização do uso dos medicamentos entre os idosos ${ }^{(15)}$; a necessidade de ampliar a visão dos profissionais de saúde para o cuidado nas instiuições de longa permanência para idosos (ILPIs) ${ }^{(16)}$; o esforço em cuidar do idoso, reconhecendo as especificidades e estimulando a independência e autonomia ${ }^{(17)}$; a necessidade da divulgação do conhecimento produzido que é um desafio para o cuidado com qualidade e cada vez mais complexo ${ }^{(18)}$. 
Frente às características próprias do envelhecimento, trabalhos que envolvam a análise, reflexão e pesquisa com a população idosa, tornam-se necessários, pois trazem contribuições para os enfermeiros, à Enfermagem, à população idosa e à sociedade como um todo ${ }^{(2)}$.

Destaca-se o número de doutores entre os pesquisadores, o que está associado ao crescente interesse dos profissionais pela temática do idoso, especialmente aqueles envolvidos com a docência ${ }^{(2)}$. Esses pesquisadores impulsionam o fomento à pesquisa, refletindo no aumento da produção científica ${ }^{(1)}$, além de conseguirem financiamentos em seus estudos, por meio de projetos oriundos nos órgãos de fomento e do ministério da saúde.

Verificou-se que a participação dos graduandos nos grupos de pesquisa é alta em relação aos demais níveis de treinamento. Anteriormente se constatou a baixa participação desses acadêmicos nos grupos de estudo e pesquisa da enfermagem, certificados pelo CNPq entre 2005 e 200710.

Nos grupos de estudo e pesquisa da área da Enfermagem, com ênfase na temática idoso evidencia-se a interdisciplinaridade dos conceitos e conhecimentos no âmbito da saúde, o que retrata o avanço na ampliação e integração do conhecimento, por meio de troca de informações e saberes, contribuindo para a construção de tecnologias, qualidade e integralidade do cuidado prestado ${ }^{(10)}$.

\section{CONCLUSÃO}

O objetivo deste estudo foi atingido e a pesquisa documental mostrou-se adequada, direcionando ao alcance do objetivo da investigação.

Como ponto positivo do estudo, destaca-se que o Diretório do CNPq apresenta-se como ferramenta facilitadora, fator relevante para o desenvolvimento efetivo desta pesquisa. Como limitação, verificou-se a desatualização de alguns grupos de pesquisa e a ausência de adequação às orientações do CNPq para o cadastro dos grupos, o que pode apresentar-se como uma lacuna quanto à fidedignidade dos dados.

Como contribuição desse estudo à Enfermagem, vislumbra-se a necessidade de estudos voltados ao idoso e a relevância da participação do enfermeiro em grupos de estudo e pesquisa, que possam contribuir para o fortalecimento e a busca de novos conhecimentos na temática investigada.

\section{Referências}

1. Kletemberg DF, Padilha MI, Gonçalves LHT, Borenstein MS, Alvarez AM, Ferreira AC. A construção histórica do conhecimento da enfermagem gerontológica no Brasil. Esc Anna Nery Rev Enferm. 2010; 14(4): 787-96.

2. Hammerschmidt KS de A, Borghi AC da S, Lenardt MH, Seima MD. Pesquisas de enfermagem em gerontologia. Cogitare Enferm. 2007; 12(2): 214-21.

3. Barbosa S de FF, Sasso GTMD, Berns I. Enfermagem e tecnologia: análise dos grupos de pesquisa cadastrados na plataforma Lattes do CNPq. Texto Contexto Enferm. 2009; 18(3): 443-8.

4. Caldas CP, Amorim AE. Os grupos de pesquisa em enfermagem no Brasil e o envelhecimento humano. Rev Cienc Saude. 2004; 23(2): 28-36.

5. Sá-Silva JR, Almeida CD, Guindani JF. Pesquisa documental: pistas teóricas e metodológicas. Rev Bras Hist Ciênc Soc. 2009; 1(1): 1-15

6. Cellard A. A análise documental. In: Poupart J. et al. A pesquisa qualitativa: enfoques epistemológicos e metodológicos. Petrópolis: Vozes; 2008, p. 295-316.

7. Conselho Nacional de Desenvolvimento Científico e Tecnológico (CNPq). Grupos de pesquisa: saiba mais. [acesso em 15 Out 2011]. Disponível em: http://dgp.cnpq.br/diretorioc/ html/faq.html

8. Prado SD, Sayd JD. A pesquisa sobre envelhecimento humano no Brasil: pesquisadores, temas e tendências. Cienc Saude Colet. 2004; 9(3): 763-72.

9. Gomes DC, Backes VMS, Lino MM, Canever BP, Ferraz F, Schveitzer MC. Produção científica em educação em enfermagem: grupos de pesquisa Rio de Janeiro e Minas Gerais. Rev Gaúcha Enferm. 2011; 32(2): 330-7.

10. Erdmann AL, Lanzoni GMM. Características dos grupos de pesquisa da enfermagem brasileira certificados pelo CNPq de 2005 a 2007. Esc Anna Nery Rev Enferm. 2008; 12(2): 316-22.

11. Coordenação de Aperfeiçoamento de Pessoal de Nível Superior (CAPES). Mestrados/Doutorados Reconhecidos. [acesso 15 Out 2011]. Disponível em: http://conteudoweb.capes.gov.br/ conteudoweb/ProjetoRelacaoCursosServlet?acao=pesquisarRegiaol es\&codigoRegiao=1\&descricaoRegiao=Norte

12. Instituto Brasileiro Geografia e Estatística (BR). [acesso 15 Out 2011]. Disponível em: http://www.ibge.gov.br/brasil_em_sintese/

13. Paskulin LMG, Aires M, Valer DB, Morais EP, Freitas IBA.

Adaptação de um instrumento que avalia alfabetização em saúde das pessoas idosas. Acta Paul Enferm. 2011; 24(2): 271-7.

14. Marin MJS, Rodrigues LCR, Druzian S, Cecílio LCO. Diagnósticos de enfermagem de idosos que utilizam múltiplos medicamentos. Rev Esc Enferm USP. 2010; 44(1): 47-52.

15. Secoli, SR. Polifarmácia: interações e reações adversas no uso de medicamentos por idosos. Rev Bras Enferm. 2010; 63(1): 136-40.

16. Santos SSC, Valcarenghi RV, Barlem ELD, Silva BT, Hammerschmidt KSA, Silva ME. Elaboração de prontuário do residente em uma instituição de longa permanência para idosos. Acta Paul Enferm. 2010; 23: 725-31.

17. Almeida ABA, Aguiar MCG. A dimensão ética do cuidado de enfermagem ao idoso hospitalizado na perspectiva de enfermeiros. Rev Eletr Enf. 2011; 13(1): 42-9.

18. Camacho ACLF, Coelho MJ. Políticas públicas para a saúde do idoso: revisão sistemática. Rev Bras Enferm. 2010; 63(2): 279-84. 